\title{
Projeto Integra: fortalecimento da participação social na agenda das políticas, serviços e tecnologias em saúde
}

\author{
Integra Project: strengthening social participation \\ in the agenda of health policies, services, and technologies
}

Silvana Nair Leite (https://orcid.org/0000-0002-5258-9684) ${ }^{1}$

Jorge Antonio Zepeda Bermudez (https://orcid.org/0000-0002-4657-0709) ${ }^{2}$

Débora Melecchi (https://orcid.org/0000-0002-4578-8589) ${ }^{3}$

Adelir Rodrigues da Veiga (https://orcid.org/0000-0001-9321-9615) ${ }^{1}$

Ana Liani Beisl Oliveira (https://orcid.org/0000-0002-6413-5727) ${ }^{2}$

Artur Custódio Moreira de Sousa (https://orcid.org/0000-0003-0995-

5404)) ${ }^{3}$

Célia Machado Gervásio Chaves (https://orcid.org/0000-0002-4210-3521) ${ }^{6}$

Fernanda Manzini (https://orcid.org/0000-0002-3047-4632) ${ }^{1}$

Lidiane Silva Dutra (https://orcid.org/0000-0003-3350-1365) ${ }^{4}$
Luísa Arueira Chaves (https://orcid.org/0000-0002-9597-3651) ${ }^{5}$

Marco Aurélio Pereira (https://orcid.org/0000-0001-8798-656X) ${ }^{3}$

Maria Eufrásia Oliveira Lima (https://orcid.org/0000-0002-08468156) ${ }^{6}$

Moysés Longinho Toniolo (https://orcid.org/0000-0003-3486-0933) ${ }^{3}$ Ronald Ferreira dos Santos (https://orcid.org/0000-0002-49051218) ${ }^{6}$

Silvânia Dantas (https://orcid.org/0000-0001-8669-7838) ${ }^{2}$

Jorge Carlos Santos da Costa (https://orcid.org/0000-0002-42990562) ${ }^{2}$
${ }^{1}$ Escola Nacional

dos Farmacêuticos, Universidade Federal de Santa Catarina. Farmácia Escola UFSC, Campus Universitário Reitor João David Ferreira Lima, Rua Delfino Conti $\mathrm{s} / \mathrm{n}$, Trindade. 88040-370 Florianópolis SC Brasil. silvana.nair.leite@ufsc.br ${ }^{2}$ Escola Nacional de Saúde Pública Sergio Arouca, Fundação Oswaldo Cruz. Rio de Janeiro RJ Brasil. ${ }^{3}$ Conselho Nacional de Saúde, Ministério da Saúde. Brasília DF Brasil.

${ }^{4}$ Secretaria de Estado da Saúde de Minas Gerais. Belo Horizonte MG Brasil.

${ }^{5}$ Universidade Federal do Rio de Janeiro. Rio de Janeiro RJ Brasil.

${ }^{6}$ Federação Nacional dos Farmacêuticos. São Paulo SP Brasil.

\begin{abstract}
The 16th National Health Conference illustrated the interest of health councils to intervene in public policies in order to guarantee the right to health technologies. The INTEGRA project (Integration of policies for Health Surveillance, Pharmaceutical Care, Science, Technology, and Innovation in Health) is a partnership among the National Health Council, the National School of Pharmacists, and the Oswaldo Cruz Foundation (Fiocruz), with support from the Pan American Health Organization (PAHO), with the goal of strengthening participation and social engagement in the theme, as well as the integration of health policies and practices within different sectors of society (social movements, health councils, and health professionals), with the various stages related to the access to medicines (research, incorporation, national production, and services) being the main theme in the context of the COVID-19 pandemic. It seeks to offer training for leadership groups in the health regions and activities with a broad national and political scope, and it hopes to establish an intersectorial and integrated network of leaders capable of acting collaboratively to defend the development of science, public policies, national sovereignty, and social control of health. Key words Pharmaceutical Care, Health Surveillance, Science, Technology and Innovation, Social Control
\end{abstract}

Resumo A $16^{a}$ Conferência Nacional de Saúde demonstrou o interesse do controle social em intervir sobre as políticas públicas a fim de garantir o direito às tecnologias de saúde. O projeto Integra - Integração das Políticas de Vigilância em Saúde, Assistência Farmacêutica, Ciência, Tecnologia e Inovação em Saúde -, nasce da parceria entre o Conselho Nacional de Saúde, a Escola Nacional dos Farmacêuticos e a Fundação Oswaldo Cruz (Fiocruz), com apoio da Organização Pan-Americana de Saúde (OPAS) com objetivo de fortalecimento da participação e engajamento social na temática e a integração das políticas e práticas de saúde em diferentes setores da sociedade (movimentos sociais, controle social e profissionais de saúde), tendo as diversas etapas relacionadas ao acesso aos medicamentos (pesquisa, incorporação, produção nacional e serviços) como mote principal, no cenário da pandemia de COVID-19. Oferta-se, neste projeto, capacitação para grupos de lideranças nas regiões de saúde e atividades de grande abrangência nacional e política. Espera-se alcançar o estabelecimento de uma rede intersetorial, integrada de lideranças capazes de atuar colaborativamente para a defesa do desenvolvimento da ciência, das políticas públicas, da soberania nacional e do controle social da saúde.

Palavras-chave Assistência farmacêutica, Vigilância em Saúde, Ciência, Tecnologia e Inovação; Controle social 


\section{Introdução}

Há três anos, a sociedade brasileira se preparava para participar da 16a Conferência Nacional de Saúde. Encontros preparatórios regionalizados culminaram com o $8^{\circ}$ Simpósio Nacional de Ciência, Tecnologia e Assistência Farmacêutica no fim do ano de 2018, no qual foram consolidadas propostas muito contundentes de defesa do acesso aos medicamentos e às tecnologias de saúde, em serviços humanizados e caracterizados pela resolutividade ${ }^{1,2}$.

Entre as estratégias indicadas já em 2018 como indispensavelmente necessárias estão a ampliação dos investimentos e da regulação para o desenvolvimento científico e tecnológico brasileiro para atender as necessidades próprias da população e dos serviços de saúde. Os elevados custos e preços dos medicamentos e outras tecnologias, as barreiras regulatórias e de propriedades intelectual ${ }^{3,4}$ foram temas que estiveram presentes nas apresentações e nas discussões. Diversas propostas para o direcionamento dos investimentos, com priorização para pesquisas em doenças negligenciadas e endêmicas e para a qualificação de serviços foram contundentemente defendidas ${ }^{2}$.

A 16a Conferência Nacional de Saúde reafirmou tais proposições, demonstrando o interesse da sociedade em compreender, discutir e intervir sobre as políticas públicas que definem as condições para a garantia do direito à saúde e do Estado democrático ${ }^{5}$.

$O$ que veio a seguir exemplifica, na infeliz realidade, a necessidade das medidas defendidas na $16^{a}$ Conferência Nacional de Saúde. A decisão política de forte desinvestimento no sistema de saúde tem levado ao abrupto colapso do SUS, agravado pela pandemia de COVID- $19^{6}$. O Brasil tornou-se um dos epicentros da pandemia da COVID-19 e está sofrendo um desastre de saúde pública $^{7}$. O vácuo de ações do governo federal para coordenar a luta contra a pandemia, os processos de desinformação generalizada, inclusive e principalmente governamental, e a falta de integração entre sociedade civil e gestores agravam a dificuldade enfrentada no país ${ }^{2}$. As condições de desigualdade da nossa sociedade, com uma enorme população em situações de extrema vulnerabilidade, são agravadas na atual situação ${ }^{7}$, culminando no que está sendo chamado de sindemia ${ }^{8}$.

É vital assegurar a viabilidade a médio e longo prazo das respostas nacionais à COVID-19 para minimizar os seus impactos sociais e sanitários prejudiciais previstos ${ }^{9}$. É preciso aprender com a experiência vivida e preparar a sociedade e o Sistema Único de Saúde (SUS) para enfrentar de forma mais harmoniosa e eficaz os desafios do futuro.

No contexto da pandemia da COVID-19, o controle social da saúde tem tido papel destacado, pelo protagonismo dos conselhos de saúde, das instituições de pesquisa e ensino e dos movimentos organizados da sociedade civil, e têm envidado esforços no sentido de informar a população, vigiar as ações das instituições públicas, provocar o estabelecimento de políticas públicas necessárias, através dos instrumentos produzidos (Resoluções, Recomendações, Notas, Cartas), e incentivar o desenvolvimento e defesa da credibilidade da ciência.

Neste contexto nasce o projeto Integra - Integração das Políticas de Vigilância em Saúde, Assistência Farmacêutica, Ciência, Tecnologia e Inovação em Saúde - numa parceria do Conselho Nacional de Saúde, Escola Nacional dos Farmacêuticos e Fundação Oswaldo Cruz (Fiocruz) com apoio da Organização Pan-americana de Saúde (OPAS). Seu objetivo final é o fortalecimento e integração das políticas e práticas de saúde em diferentes setores da sociedade (movimentos sociais, controle social e profissionais de saúde). Para tanto, propõe a oferta de capacitação em atividades conjuntas de grupos de lideranças nas regiões de saúde e atividades de grande abrangência social. Espera-se alcançar o estabelecimento de uma rede intersetorial, integrada, de lideranças capazes de atuar colaborativamente para a defesa do desenvolvimento da ciência, das políticas públicas, da soberania nacional e do controle social da saúde.

Para tanto, algumas das bases norteadoras do projeto são descritas a seguir.

\section{A intersetorialidade no SUS}

A Constituição Federal de 1988 introduziu significativos avanços ao ordenamento legal brasileiro, ampliando direitos, corrigindo iniquidades e assegurando a participação popular na elaboração das políticas públicas. O Sistema Único de Saúde (SUS) surge neste contexto, inovando ao adotar como princípios as ideias de complementariedade e intersetorialidade em contraponto a herança histórica de políticas públicas sanitárias centralizadas e verticalizadas. A gestão, planejamento, controle e avaliação aproximou-se mais dos usuários, reconhecendo-os como cidadãos e portadores de direitos fundamentais, não só pela instituição efetiva da participação comunitária 
nos Conselhos e Conferencias, mas também no aprimoramento das Ouvidorias e Auditorias do SUS. Deste modo, a descentralização proposta na organização do SUS possibilitou uma gestão mais participativa e democrática ${ }^{10}$.

No entanto, em observância ao processo de implementação dos serviços públicos após a Constituição Federal de 1988, identifica-se a temática da intersetorialidade e da articulação como elementos ainda desafiadores, pois, embora muitas vezes as organizações estejam descentralizadas, as mesmas ainda se encontram setorializadas, sem que haja uma busca integrada do verdadeiro atendimento das demandas. Assim, a necessidade da intersetorialidade tornou-se notória à medida que não se observava a eficiência, a efetividade e a eficácia esperadas na implementação das políticas setoriais, primordialmente no que se refere ao atendimento das demandas da população e aos recursos disponibilizados para execução de tais ações ${ }^{10,11}$.

Pode-se definir a intersetorialidade como consequência e produto da articulação de diversos saberes, apreendida como um novo processo na dinâmica governamental para o alcance de resultados integrados perante o enfrentamento de problemas complexos, com ações voltadas aos interesses coletivos, permeados pela participação da sociedade civil, e que têm como resultado a maior eficiência da gestão política e dos serviços prestados $^{11-13}$.

Em relação à saúde, a interlocução específica entre as políticas públicas é problematizada em função dos desafios comuns que as reúnem em torno da complexa determinação social das condições de vida e saúde da população. Além disto, tais políticas compartilham um desenho de implementação territorializado, articulado por princípios de hierarquização e regionalização, o que resulta no fato de que estão em um mesmo território, atendendo a mesma população, com objetivos específicos diferentes e, ainda assim, indissociáveis ${ }^{13}$. A intersetorialidade incorpora a ideia de integração, de território, de equidade, enfim dos direitos sociais; é uma nova maneira de abordar os problemas, uma vez que, observase que cada política social encaminha a seu modo uma solução, sem considerar o cidadão na sua totalidade e nem a ação das outras políticas sociais, que também estão buscando a melhoria da qualidade de vida. A intersetorialidade é defendida há muito tempo como uma alternativa para solucionar tais problemas, pois indica uma perspectiva conjunta de problemas e possíveis soluções, através da otimização dos recursos escas- sos, e considerando a complexidade da realidade social, que inevitavelmente não ficará apenas na implementação de uma única política pública ${ }^{14}$. No entanto, ainda hoje verifica-se sua pouca implementação na área da saúde.

A intersetorialidade não é algo que ocorre espontânea e naturalmente nas instituições públicas sem provocar resistência e exigir mudanças substanciais no modelo organizacional ${ }^{12,15}$. Uma mudança paradigmática na direção intersetorial não pressupõe preterir as disciplinas em suas singularidades, e sim ensejar um processo comunicacional, não só para ressignificar as práticas na gestão pública, mas também enriquecer a compreensão das diferenças de saberes. A setorialidade, portanto, não é necessariamente suprimida pela intersetorialidade, já que os domínios disciplinares devem ser sintetizados através da comunicação ${ }^{11,16,17}$.

Trabalhar de modo intersetorial significa superar a fragmentação do conhecimento e da prática envolvendo, também, a população que vivencia o problema na busca de soluções compartilhadas. Nisso reside seu papel de superar fragmentações, não só na estrutura do aparato estatal, mas também no campo do conhecimento, superando práticas assistencialistas e de gestão fragmentárias. Além disso, a intersetorialidade é um canal para lidar com as resistências dos trabalhadores; garantir maior participação e controle social; e por fim, realizar a gestão, avaliação e o monitoramento das ações intersetoriais com vistas ao alcance de maior integralidade e equidade nas ofertas públicas das políticas em foco ${ }^{11-13}$.

\section{O controle social na saúde e as políticas públicas em questão}

O ciclo de uma política pública abrange as etapas de formação de agenda, formulação da política, tomada de decisão, implementação e avaliação. Os processos pré-decisórios, anteriores à formalização da política, consistem em enumerar os problemas e definir as alternativas possíveis de intervenção. Diferentes atores estão envolvidos no processo de entendimento e definição do problema, na fase da agenda, que definirá todo o debate posterior no processo de formulação da política ${ }^{18}$.

A sociedade civil, no Brasil, participa da formulação e controle das políticas públicas de saúde através do controle social. Os Conselhos e as Conferências de Saúde, regulamentados pela Lei Orgânica de Saúde n ${ }^{\circ} .142 / 90$, além de outros espaços institucionalizados no arcabouço jurídico do SUS, são meios utilizados para exercer o 
controle social a partir do diálogo com diferentes segmentos de usuários, trabalhadores e prestadores de serviço ${ }^{19}$.

Apesar de ainda não ocupar de forma efetiva essa participação $0^{19}$, o controle social amadureceu desde a criação do SUS, estabelecendo maior representatividade e influência sobre as políticas públicas $^{20}$. Evidências demonstram os limites e potencialidades dos Conselhos Municipais de Saúde, ressaltando a importância da capacitação dos conselheiros e que, para muitos dos autores pesquisados, a pouca participação de alguns conselheiros deve-se ao desconhecimento que estes têm de seu próprio papel e de como exercê-lo ${ }^{21} \mathrm{e}$ do desconhecimento das políticas que orientam as ações e serviços públicos de saúde.

A Política Nacional de Ciência, Tecnologia e Inovação em Saúde (PNCTIS), a Política Nacional de Assistência Farmacêutica (PNAF) e a Política Nacional de Vigilância em Saúde são oriundas das lutas dos movimentos sociais organizados, devido às necessidades de saúde da população. Portanto, pode-se afirmar que nascem dos segmentos socialmente representativos da sociedade e buscam trazer a equidade e a racionalidade no acesso e atenção à saúde ${ }^{4}$.

A integração destas áreas da saúde: ciência, tecnologia, assistência farmacêutica e vigilância em saúde constitui elemento central dos Planos de Trabalho do triênio 2019/2022 das Comissões Intersetoriais do Conselho Nacional de Saúde que se relacionam a estes temas, sendo as Comissões de Ciência, Tecnologia e Assistência Farmacêutica, de Vigilância em Saúde, e de Atenção à Saúde das Pessoas com Patologias.

O debate com a população sobre as políticas PNCTIS, PNAF e Vigilância em Saúde é fundamental para que estas alcancem maior envolvimento de diferentes setores da sociedade civil no controle social e possam refletir os interesses do conjunto da sociedade brasileira. $\mathrm{O}$ maior conhecimento destas políticas e a percepção de como elas se integram desencadeará maior envolvimento da população na formação da agenda de políticas públicas assertivas e baseadas na compreensão mais nítida sobre os determinantes e condicionantes para a promoção da saúde no Brasil.

\section{A pandemia como caso concreto para identificação das fragilidades $e$ potencialidades do sistema}

A pandemia da COVID-19 vem desafiando os sistemas de saúde do mundo todo, evidenciando, sobremaneira, suas fragilidades e explicitando a necessidade de uma maior integração e estruturação sistêmica das diretrizes do SUS expressas no artigo 198 e a urgência da apropriação pela sociedade do conceito ampliado de saúde, constatado na constituição de 1988.

Esta emergência sanitária trouxe a necessidade de se refletir e implementar novas estratégias de organização da oferta de serviços nos territórios; planejamento de compras de insumos para a assistência à saúde, em um ambiente de aumento de demanda e escassez de oferta; captação de recursos humanos para a realização desses serviços e; sustentando todas essas ações, políticas sociais e econômicas que garantissem a redução das vulnerabilidades socioeconômicas de nossa população. Para que essas ações fossem efetivas no enfrentamento da pandemia, a articulação entre os entes federados e, das diversas políticas públicas eram cruciais para o sucesso dessas estratégias. Infelizmente, não foi isso que se verificou, principalmente no compartilhamento do planejamento e na tomada de decisão entre as instâncias gestoras e o controle social do SUS.

Logo no início da pandemia, o principal problema foi garantir uma tomada de decisão estratégica e com prioridades sobre as compras de testes diagnósticos, seus insumos e Equipamentos de Proteção Individual (EPI) ${ }^{22}$. No mesmo momento, também foi importante pensar na organização do sistema de saúde para atender a demanda de atendimentos à saúde da população, tanto por sintomas da COVID-19, como na manutenção da atenção para outros problemas de saúde naturais e já incorporados na rotina dos serviços. Foi necessário pensar na reestruturação dos processos de trabalho da atenção primária à saúde, vigilância em saúde, urgências e emergências e unidades de internação hospitalar, onde os processos eletivos foram paralisados diante da emergência em saúde relacionada a epidemia nova.

A despeito de diversas evidências internacionais que demonstravam a importância da estruturação da atenção primária no rastreamento e controle de infecções por COVID-19 no território, testagem em massa, acompanhamento e isolamento dos casos positivos e seus contatos ${ }^{23-25}$, assim como a ativação correta dos serviços de imunização ligados à atenção básica; pouco foi feito ou implementado nesse sentido. Por um outro lado, o que se observou foi a preferência por estratégias como a compra de medicamentos sem eficácia terapêutica ${ }^{26}$; e a abertura de hospitais de campanha e/ou de novos leitos hospitalares que, embora necessárias para o atendimento da popu- 
lação, quando implementadas de maneira isolada e desarticulada de outras ações, não são capazes nem de evitar a sobrecarga das unidades hospitalares $^{27}$.

De maneira transversal a todos os problemas de organização da rede de atenção à saúde nos territórios, ficou claro a fragilidade da base produtiva nacional em ofertar os insumos necessários ao combate da pandemia. A forte dependência do Brasil à importação de fármacos, medicamentos e demais insumos para a saúde ${ }^{28-30}$ comprometeram nossa capacidade de resposta à pandemia devido a dificuldades para a compra de testes diagnósticos e seus insumos, EPIs, equipamentos e medicamentos necessários para $\mathrm{o}$ cuidado em saúde.

A pandemia também demonstrou a importância do SUS para os brasileiros que, mesmo com todas as fragilidades, continuou a atender a seus cidadãos ao longo de toda a pandemia, inclusive àqueles que têm cobertura do sistema privado suplementar e viram seu direito negado pelas operadoras de saúde. Sem o SUS, com certeza a situação seria pior. No entanto, é importante retomar, de forma prática, as diretrizes legais dos SUS, de modo que possamos pensar as políticas de saúde de modo ampliado, participativo e articulado às demais políticas públicas. Para isso, $\mathrm{e}$ em homenagem aos milhares de mortos acometidos pela pandemia, devemos usar os problemas ocorridos como lição e utilizá-los para melhoria de nosso sistema de saúde, implementando estratégias e ações que possam reduzir essas fragilidades e garantir a missão do SUS em nosso país.

Assim, apoiado nos princípios constitucionais, inspirado pela experiência da construção do $8^{\circ}$ SNCTAF $^{2}$ e impulsionado pelas questões evidenciadas pela pandemia do Coronavírus que esse projeto se propõe a realizar o debate integrando as seguintes áreas da saúde: ciência, tecnologia, assistência farmacêutica e vigilância em saúde, com mobilização social, proposição de ações políticas e a formação de lideranças nas políticas públicas de saúde que com ela se relacionam intrinsecamente. A Proposta visa trabalhar a integralidade e a integração das políticas públicas já citadas, com fundamento nas necessidades descritas pelo controle social da saúde, fruto de extensos e ricos debates, a partir de processos de conferências que deliberam sobre os seus temas específicos.

\section{Metodologia proposta}

\section{O projeto Integra}

A metodologia proposta no Projeto Integra visa proporcionar condições para uma formação mais sólida e estruturada de lideranças preparadas teórico-conceitualmente para desenvolver ações relacionadas ao tema proposto, com criação de vínculos e compromissos mais perenes entre os participantes e entre estes e as instituições de saúde locais e nacionais. Além disso, propõe o desenvolvimento de atividades de educação e mobilização social de forma aberta e abrangente, a construção de referenciais para a integração das políticas e a proposição concreta de ações políticas neste sentido. Por este motivo, propõe uma construção em fases (Figura 1):

Primeira fase - Capacitar lideranças: Formação de lideranças regionais sobre as políticas públicas em questão

Segunda fase - Sensibilizar e engajar a sociedade e as instituições: Realização de encontros regionais preparatórios para o 9० Simpósio $\mathrm{Na}$ cional de Ciência, Tecnologia, Vigilância em Saúde e Assistência Farmacêutica.

Terceira fase - Consolidar propostas: Realização do $9^{\circ}$ Simpósio e aprovação do relatório com o referencial construído.

Quarta fase - Promover ação política: Realização de debates e audiências públicas na Câmara e no Senado Federal, nas Assembleias Legislativas, nas Câmaras Municipais, nas Universidades e nas Coordenações Regionais da Fiocruz (onde houver) para apresentação do Relatório Final aprovado no 9o Simpósio.

Para a primeira fase se desenvolvem estratégias e recursos interativos para o reconhecimento, análise e reflexão sobre os eixos principais (Ciência, Tecnologia e Inovação em saúde, Assistência Farmacêutica, Vigilância em Saúde e Controle Social), sobre os quais se pretende aprofundar no $9^{\circ}$ Simpósio. A metodologia para esta fase consiste em atividades síncronas e assíncronas em grupos de até 20 participantes, sob a tutoria de um profissional experiente, dedicado a esta atividade. Cada grupo de participantes estará engajado com estas atividades por um período de dois meses e terá frequência monitorada.

A capacitação das lideranças é realizada com base na formação de competências que envolvem conhecimento, habilidades e atitudes. As competências necessárias para que os líderes atuem na integração das políticas em Ciência, Tecnologia e Inovação em saúde (CT\&I), Vigilância em Saú- 


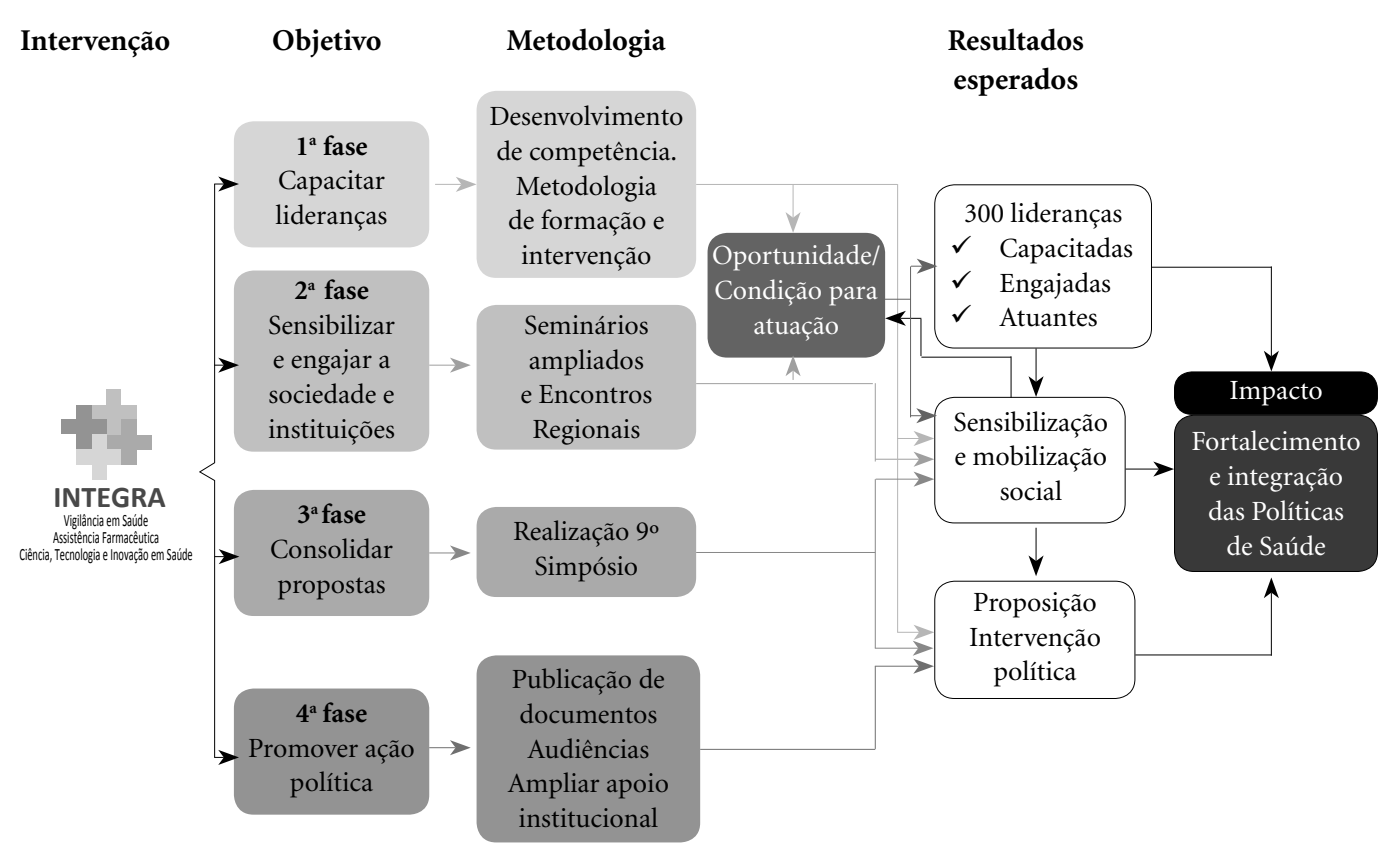

Figura 1. Fases de desenvolvimento do Projeto Integra.

Fonte: Elaborado pelos autores.

de (VS), a Assistência Farmacêutica (AF) junto às instituições e aos movimentos sociais foram definidas com base nos objetivos propostos no Projeto Integra e em oficinas de consenso com a participação de integrantes do Conselho Nacional de Saúde, da Fiocruz e da Escola Nacional dos Farmacêuticos, e estão descritas na Figura 2.

A formação, em atividades de 40 horas, está fundada em casos baseados em experiências reais e ou verossimilhanças a situações reais que tiveram, ou têm, algum impacto social (relativas a pacientes com COVID-19, por exemplo) sendo estes casos ferramentas de sensibilização e disparadores do debate. Em todos os casos, a narrativa integra parte de casos concretos muito próximos da realidade vivida pelas pessoas na atualidade, e desenvolve-se para alcançar sua relação com as políticas que pretende-se abordar: Ciência e Tecnologia e inovação em saúde (PNCTIS), Assistência Farmacêutica (PNAF) e Vigilância em Saúde (PNVS). A descrição dos conteúdos e atividades está apresentada no Quadro 1.

Após completada esta primeira fase para todos os participantes em cada uma das regiões, será procedida a $2^{\mathrm{a}}$ fase, com a organização e realização dos Encontros Regionais Preparatórios para o 90 Simpósio. A metodologia proposta para os encontros baseia-se na experiência de metodologia ativa participativa com a apresentação de situações-problema em forma de "casos" com narrativas reais, fictícias ou adaptadas da realidade, à semelhança da proposta adotada anteriormente nos Encontros Preparatórios para o $8^{\circ}$ Simpósio ${ }^{2}$ e da formação das lideranças na fase 1 do Projeto Integra. Esta fase será realizada de forma virtual e presencial (quando as condições epidemiológicas permitirem). As proposições construídas em cada encontro subsidiarão a construção do $9^{\circ}$ Simpósio Nacional.

Para a fase do $9^{\circ}$ Simpósio Nacional, lideranças de todas as regiões e das principais instituições nacionais serão convidadas a debater as proposições sistematizadas e definir as prioritárias, que deverão compor o planejamento de ações do controle social em todos os níveis de gestão. $\mathrm{Na}$ fase final, a rede de colaboração deve estar estabelecida e consolidada para viabilizar perenemente a articulação e ação coordenada de lideranças no tema do projeto.

Considerando a importância da avaliação de estratégias educacionais para orientar a tomada de decisões e futuros investimentos em capacitações/formações $\mathrm{e}^{31,32}$, o projeto terá seus resultados avaliados em cada fase. 


\section{Desenvolvimento de competências}

Fase 1

\section{Objetivo: Capacitar lideranças}

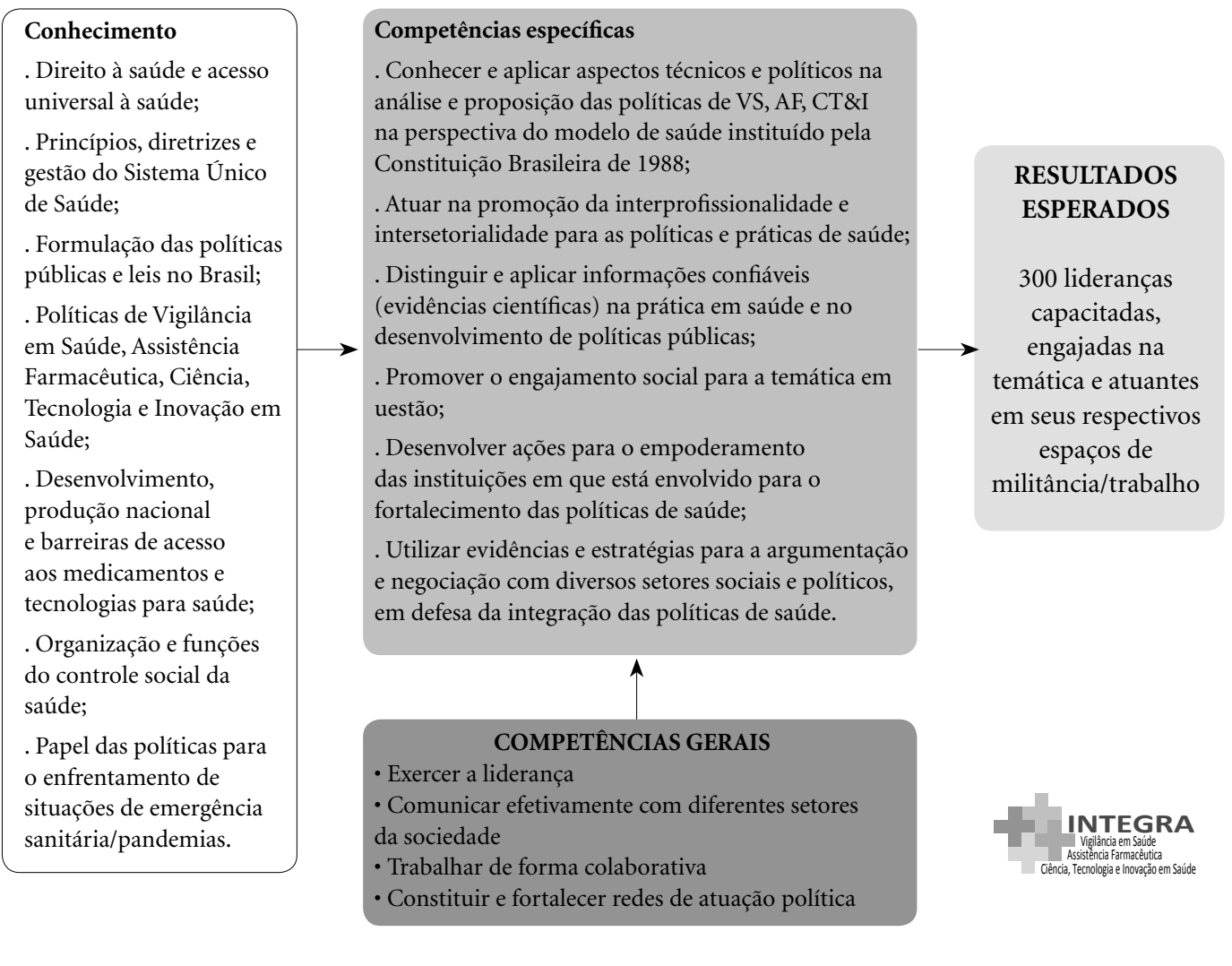

Figura 2. Desenvolvimento de competências na formação de lideranças no Projeto Integra - Fase 1.

Fonte: Elaborado pelos autores.

Quadro 1. Temas, conteúdos e atividades para o curso de formação de lideranças - Projeto Integra - Fase 1.

\begin{tabular}{|l|l|l|l|}
\hline Semana & \multicolumn{1}{|c|}{ Tema } & \multicolumn{1}{c|}{ Objetivo Geral } & \multicolumn{1}{c|}{ Atividade de intervenção } \\
\hline $\mathbf{1}$ & $\begin{array}{l}\text { Direito à saúde, } \\
\text { acesso universal e } \\
\text { equidade }\end{array}$ & $\begin{array}{l}\text { Reconhecer a saúde enquanto direito fun- } \\
\text { damental, com acesso universal e equânime }\end{array}$ & $\begin{array}{l}\text { Mapeamento sociodemográfico } \\
\text { do território: } \\
\text { Identificação de fontes de dados; } \\
\text { Reconhecimento das instituições } \\
\text { de pesquisa e desenvolvimento } \\
\text { na região }\end{array}$ \\
\hline $\mathbf{2}$ & $\begin{array}{l}\text { Produção e acesso } \\
\text { às vacinas }\end{array}$ & $\begin{array}{l}\text { Compreender como as políticas de Vigilân- } \\
\text { cia em Saúde (PNVS), de Ciência Tecnolo- } \\
\text { gia e Inovação em Saúde (PNCTIS) e de As- } \\
\text { sistência Farmacêutica (PNAF) contribuem } \\
\text { para a garantia do acesso à vacinação }\end{array}$ & $\begin{array}{l}\text { Estratégia de vacinação adotada } \\
\text { pelo município }\end{array}$ \\
\hline $\mathbf{3}$ & $\begin{array}{l}\text { Testagem e rastre- } \\
\text { amento de conta- } \\
\text { tos na pandemia } \\
\text { de COVID-19 }\end{array}$ & $\begin{array}{l}\text { Reconhecer o potencial do Brasil para } \\
\text { implementar medidas de combate a pande- } \\
\text { mias e em desenvolver tecnologias em saúde } \\
\text { por meio da efetivação das Políticas de Ci- } \\
\text { ência, Tecnologia e Inovação, de Vigilância } \\
\text { em Saúde e de Assistência Farmacêutica }\end{array}$ & $\begin{array}{l}\text { Estratégia de vigilância em saúde, } \\
\text { considerando COVID-19 e outras } \\
\text { doenças transmissíveis }\end{array}$ \\
\hline
\end{tabular}


Quadro 1. Temas, conteúdos e atividades para o curso de formação de lideranças - Projeto Integra - Fase 1.

\begin{tabular}{|l|l|l|l|}
\hline Semana & \multicolumn{1}{|c|}{ Tema } & \multicolumn{1}{|c|}{ Objetivo Geral } & \multicolumn{1}{c|}{ Atividade de intervenção } \\
\hline $\mathbf{4}$ & $\begin{array}{l}\text { Informações } \\
\text { confiáveis em } \\
\text { saúde }\end{array}$ & $\begin{array}{l}\text { Compreender a importância do uso } \\
\text { de evidências científicas no uso de } \\
\text { medicamentos, de vacinas e de outros } \\
\text { produtos na área da saúde }\end{array}$ & $\begin{array}{l}\text { Fontes de Informações confiáveis } \\
\text { em saúde e informações para } \\
\text { acesso aos medicamentos no } \\
\text { território }\end{array}$ \\
\hline $\mathbf{5}$ & $\begin{array}{l}\text { Desabastecimento } \\
\text { de medicamentos }\end{array}$ & $\begin{array}{l}\text { Refletir sobre as relações das políticas } \\
\text { (PNAF e PNCTIS) sobre a situação do } \\
\text { desabastecimento de medicamentos na } \\
\text { pandemia }\end{array}$ & $\begin{array}{l}\text { Organização para o acesso a } \\
\text { medicamentos no território }\end{array}$ \\
\hline $\mathbf{6}$ & $\begin{array}{l}\text { Atenção Básica } \\
\text { e a vigilância em } \\
\text { saúde }\end{array}$ & $\begin{array}{l}\text { Compreender a centralidade da Atenção } \\
\text { Básica, e sua interrelação territorial com a } \\
\text { vigilância em saúde }\end{array}$ & $\begin{array}{l}\text { Análise das fragilidades e } \\
\text { potencialidades na organização da } \\
\text { atenção primária à saúde no seu } \\
\text { território }\end{array}$ \\
\hline $\mathbf{7}$ & $\begin{array}{l}\text { Inovação e } \\
\text { incorporação de } \\
\text { medicamentos }\end{array}$ & $\begin{array}{l}\text { Compreender a importância da inovação } \\
\text { de medicamentos e como ocorre sua } \\
\text { incorporação no SUS }\end{array}$ & $\begin{array}{l}\text { Exercício de priorização de } \\
\text { problemas na realidade viven- } \\
\text { ciada }\end{array}$ \\
\hline $\mathbf{8}$ & $\begin{array}{l}\text { Papel da Anvisa e } \\
\text { nua importância } \\
\text { PNCTIS, PNAF e } \\
\text { PNVS }\end{array}$ & $\begin{array}{l}\text { Compreender como a Anvisa contribui para } \\
\text { a implementação e articulação das PNVS, } \\
\text { PNCTIS e PNAF no dia-a-dia da população }\end{array}$ & $\begin{array}{l}\text { Elaboração de propostas e } \\
\text { mobilização de recursos para o } \\
\text { território }\end{array}$ \\
\hline
\end{tabular}

Fonte: Elaborado pelos autores.

\section{Um convite para o engajamento na defesa da saúde}

A educação, a motivação e o preparo da população para qualificar a participação no controle social, no pensar e agir no coletivo, para comunicar com todos os setores e para enfrentar os desafios cada vez maiores, são investimentos para uma sociedade mais democrática e saudável.

A defesa intransigente do SUS como política pública de resgate da cidadania e do direito social à saúde é uma necessidade premente. A credibilidade na ciência e nas políticas públicas está em crise e traz impactos profundos para população brasileira. A capacidade de discernir informações, construir pontes de colaboração e comunicar adequadamente são competências que precisam ser desenvolvidas por lideranças dos diferentes setores da sociedade, para que possam agir de forma coordenada e mais eficiente - o que este ambicioso projeto pretende promover. 


\section{Colaboradores}

SN Leite atuou na concepção, redação do artigo e revisão crítica. JAZ Bermudez atuou na concepção, administração do projeto e revisão do texto. CMG Chaves, D Melecchi, ACM Sousa, ML Toniolo, MA Pereira, RF Santos, S Dantas, JCS Costa trabalharam captação de recursos e coordenação do projeto. AR Veiga e MEO Lima atuaram na assessoria do projeto. LS Dutra, LA Chaves, ALB Oliveira, F Manzini atuaram na redação do artigo e revisão crítica.

\section{Agradecimentos}

Às Instituições parceiras neste projeto, à equipe de organização e produção, e aos tutores do projeto Integra.

\section{Referências}

1. Associação Brasileira de Saúde Coletiva (ABRASCO). Carta do Rio de Janeiro, 29 jul. 2018. [acessado 2021 jun 22]. Disponível em: https://www.abrasco.org.br/ site/eventos/congresso-brasileiro-de-saude-coletiva/ carta-do-rio-de-janeiro/35707/

2. Leite SN, Manzini F, Veiga A, Lima MEO, Pereira MA, Araujo SQ, Santos RF, Bermudez JAZ. Ciência, Tecnologia e Assistência Farmacêutica em pauta: contribuições da sociedade para a $16^{\mathrm{a}}$ Conferência Nacional de Saúde. Cien Saude Colet 2018; 23(12):4259-4268.

3. Bermudez JAZ, Esher A, Osorio-de-Castro CGS, Vasconcelos DMM, Chaves GC, Oliveira MA, Silva RM, Luiza VL. Assistência Farmacêutica nos 30 anos do SUS na perspectiva da integralidade. Cien Saude Colet 2018; 23(6):1937-1949.

4. Bermudez JAZ, Barros MBA. Profile of access and use of medicines in the Brazilian population - contributions and challenges of PNAUM - Household Survey. Rev Saude Publica 2016; 50(Supl. 2):2s.

5. Conselho Nacional de Saúde. Relatório Consolidado da $16^{a}$ Conferência Nacional de Saúde, ago. 2019. [acessado 2021 jun 22]. Disponível em: https://conselho.saude.gov.br/16cns/assets/files/relatorios/Relatorio_Nacional_Consolidado.pdf

6. Andrade RO. Covid-19 is causing the collapse of Brazil's national health service. BMJ 2020; 370:m3032.

7. Lotta G, Wenham C, Nunes J, Pimenta DN. Community health workers reveal COVID-19 disaster in Brazil. Lancet 2020; 396(10248):365-366.

8. Centro de Estudos Estratégicos da Fiocruz (CEE). Covid-19 não é pandemia, mas sindemia: o que essa perspectiva científica muda no tratamento. Rio de Janeiro: CEE; 2020. [acessado 2021 jun 22]. Disponível em https://cee.fiocruz.br/?q=node/1264

9. Amimo F, Lambert B, Magit A, Hashizume M. A review of prospective pathways and impacts of COVID-19 on the accessibility, safety, quality, and affordability of essential medicines and vaccines for universal health coverage in Africa. Globalization $\mathrm{He}$ alth 2021; 17(1).

10. Monnerat GL, Souza RG. Da Seguridade Social à intersetorialidade: reflexões sobre a integração das políticas sociais no Brasil. Revista Katálysis 2011; 14(1):41-49.

11. Inojosa RM. Sinergia em políticas e serviços públicos: desenvolvimento social com intersetorialidade. $\mathrm{Ca}$ dernos Fundap 2001; 22:102-110.

12. Junqueira LAP. Intersetorialidade, transetorialidade e redes sociais na saúde. Revista de Administração Pública 2000; 34(6):35-45.

13. Nascimento S. Reflexões sobre a intersetorialidade entre as políticas públicas. Serviço Social \& Sociedade 2010; 101:95-120.

14. Junqueira LAP. A gestão intersetorial das políticas sociais e o terceiro setor. Saude Soc 2004; 13(1):25-36.

15. Pereira KYL, Teixeira SM. Redes e intersetorialidade nas políticas sociais: reflexões sobre sua concepção na política de assistência social - Networks and intersectoriality in social policies: reflections on their concept on Social assistance policy. Textos \& Contextos (Porto Alegre) 2013; 12(1):114-127. 
16. Lima EC, Vilasbôas AL. Q. Implantação das ações intersetoriais de mobilização social para o controle da dengue na Bahia, Brasil. Cad Saude Publica 2011; 27(8):1507-1519.

17. Schutz F, Mioto RCT. Intersetorialidade e política social: subsídios para o debate. Soc Debate 2010; 16(1):59-75.

18. Capella ACN. Formulação de Políticas Públicas. Brasília: Enap; 2018.

19. Rolim LB, Cruz RSBLC, Sampaio KJAJ. Participação popular e o controle social como diretriz do SUS: uma revisão narrativa. Saude Debate 2013; 37(96):139-147.

20. Lima DF, Lima LA. O controle social no Sistema Único de Saúde: um olhar crítico à Resolução nº 453/2012. Saude Debate 2017; 41(115):1168-1176.

21. Gaedtke KM, Grisotti M. os conselhos municipais de saúde: uma revisão da literatura sobre seus limites e potencialidades. Política \& Sociedade 2011; 10(19):115-138.

22. Vedovato TG, Andrade CB, Santos DL, Bitencourt SM, Almeida LP, Sampaio JFS. Trabalhadores(as) da saúde e a COVID-19: condições de trabalho à deriva? Rev Bras Saude Ocup 2021; 46:e1.

23. Johanna N, Citrawijaya H, Wangge G. Mass screening vs lockdown vs combination of both to control COVID-19: A systematic review. J Public Health Res 2020; 9(4):2011.

24. Ng Y, Li Z, Chua YX, Chaw WL, Zhao Z, Er B, Pung R, Chiew CJ, Lye DC, Heng D, Lee VJ. Evaluation of the Effectiveness of Surveillance and Containment Measures for the First 100 Patients with COVID-19 in Singapore - January 2-February 29, 2020. MMWR 2020; 69(11):307-311.

25. Rong XM, Yang L, Chu H, Fan M. Effect of delay in diagnosis on transmission of COVID-19. Mathematical Biosciences and Engineering 2020; 17(3):2725-2740.

26. Paim NA, Gnatta D. Drug Purchases's analysis for COVID-19 treatment in Rio Grande do Sul. Revista Brasileira de Farmácia Hospitalar e Serviços de Saúde 2021; 12(2):554.

27. Fundação Oswaldo Cruz (Fiocruz). Boletim Observatório Covid-19: Boletim Extraordinário. Rio de Janeiro: Fiocruz; 2021 [acessado 2021 maio 19]. Disponível em: https://portal.fiocruz.br/sites/portal.fiocruz.br/files/documentos/boletim_extraordinario_2021-marco -16-red-red-red.pdf
28. Fernandes DRA, Gadelha CAG, Maldonado JMSV. Vulnerabilidades das indústrias nacionais de medicamentos e produtos biotecnológicos no contexto da pandemia de COVID-19. Cad Saude Publica 2021; 37(4):e00254720.

29. Gadelha CAG, Kamia FD, Moreira JDD, Montenegro KBM, Safatle LP, Nascimento MAC. Dinâmica global, impasses do SUS e o CEIS como saída estruturante da crise7. Cadernos do Desenvolvimento 2021; 16(28):281-302.

30. Rodrigues PHA, Costa RDF, Kiss C. A evolução recente da indústria farmacêutica brasileira nos limites da subordinação econômica. Physis 2018; 28(1):e280104.

31. Rouse D. (NICK). Employing Kirkpatrick's Evaluation Framework to Determine the Effectiveness of Health Information Management Courses and Programs. Perspect Health Inform Manage 2011; 8 (n. Spring):1c.

32. Ruggeri K, Farrington C, Brayne C. A global model for effective use and evaluation of e-learning in health. Telemed J E-Health 2013; 19(4):312-321.

Artigo apresentado em 13/09/2021

Aprovado em 16/09/2021

Versão final apresentada em 18/09/2021

Editores-chefes: Maria Cecília de Souza Minayo, Romeu Gomes, Antônio Augusto Moura da Silva 